\title{
Formas de perder-se na floresta: Chapeuzinho Vermelho e as experiências narrativas e cognitivas nos aplicativos
}

Ways to get lost in the woods: Little Red Riding Hood and the narrative and cognitive experiences in apps

$\underline{\underline{T h a l e s ~ E s t e f a n i \mathrm{x}}}$

1 Doutorando do Programa de Doutoramento em Estudos Avançados em Materialidades da Literatura (bolsista da Fundação para a Ciência e a Tecnologia, Portugal); investigador associado ao Centro de Literatura Portuguesa da Faculdade de Letras da Universidade de Coimbra. E-mail: thales. chaun@gmail.com 
RESUMO: A partir de uma pesquisa no catálogo da Google Play, determinam-se cinco aplicativos exemplares que recontam a história de Chapeuzinho Vermelho para serem analisados de acordo com suas características semióticas, estratégias de interação e estruturas narrativas. O objetivo da análise é demonstrar, a partir das teorias sobre a cognição incorporada e distribuída, como a materialidade formal de tais aplicativos pode resultar em experiências narrativas únicas por formarem sistemas cognitivos diferentes e ampliarem sobremaneira a forma de contar a história da Chapeuzinho Vermelho.

PALAVRAS-CHAVE: Chapeuzinho Vermelho; Aplicativo literário; Narrativa; Interface; Cognição.

ABSTRACT: From a search of the Google Play catalog, five exemplary apps that tell the story of Little Red Riding Hood are determined. These applications are analyzed according to their semiotic characteristics, interaction strategies and narrative structures. The purpose of the analysis is to demonstrate, from the theories of embodied and distributed cognition, how the formal materiality of such applications can result in unique narrative experiences by forming different cognitive systems and greatly broadening the storytelling of Little Red Riding Hood.

KEYWORDS: Little Red Riding Hood; Literary app; Narrative; Interface; Cognition. 


\section{Uma floresta extensa e labiríntica}

Aplicativos de storytelling para crianças, com as mais diversas interfaces e características, multiplicam-se nas lojas virtuais. O Chapeuzinho Vermelho, por tratar-se de um conto há muito tempo pertencente ao cânone da literatura infantil mundial, não deixaria de ser também absorvido e recontado por esse meio narrativo.

Esses aplicativos para smartphones e tablets que têm como função principal transmitir histórias são por vezes denominados book apps (SARGEANT, 2015; STICHNOTHE, 2014). Eles diferem-se bastante dos genericamente chamados e-books, compreendidos como "electronic representation of a book" (GARRISH, 2011 apud SARGEANT, 2015, p. 457). Um e-book é um tipo de arquivo digital (como Mobi, $\mathrm{AZW}$, ePub) suportado por uma plataforma de e-reader específica ou softwares de leitura dedicados a esse tipo de arquivo. Já os book apps são softwares de storytelling independentes, aplicativos programados para serem executados pelos dispositivos computacionais portáteis aos quais se destinam (aqueles que operam com sistema Android ou $i O S$, por exemplo). Esses aplicativos normalmente possibilitam a experiência da transmissão de histórias individualizadas, amplamente influenciadas por diferentes mídias, interatividade e diversas formas de organização do conteúdo.

Em trabalho anterior, o autor deste artigo utilizou o termo book app para identificar o objeto de estudo (ESTEFANI, 2018). Em tal ocasião, a análise dos aplicativos dava enfoque especificamente sobre a maneira como as obras apresentavam referências formais ao códice, especialmente o livro ilustrado (picturebook). Entretanto, ao considerar os exemplos de aplicativos analisados neste artigo, é prudente repensar os termos empregados anteriormente. Aline Frederico sugere denominar os artefatos pertencentes a essa subcategoria da literatura eletrônica como literary apps, visto que neles os textos "explore the features and affordances of digital media through a programmed software, an application or app, to convey a literary work” (FREDERICO, 2017, p. 45). Essa denominação, que a pesquisadora esclarece já ter sido empregada anteriormente por Ayoe Quist Henkel $(2016)^{2}$, parece a mais adequada para

2 HENKEL, Ayoe Quist. Exploring the materiality of literary apps for children. In: Children's Literature in Education: 49 (3): 338-355, 2018 (first online: nov, 2016). 
definir os aplicativos analisados neste artigo: aplicativos de storytelling cujas características os conectam à esfera do literário, mesmo que, por vezes, eles prescindam de quase qualquer recurso formal do livro impresso.

A fim de organizar um corpus para analisar os principais tipos de aplicativos que recontam o conto de Chapeuzinho Vermelho, foi realizado um levantamento a partir dos títulos disponíveis na Google Play Store, loja que comercializa aplicativos para dispositivos com sistema operacional Android. Foram realizadas duas incursões para levantamento dos títulos na loja, tanto por meio do website - acessado através do navegador Google Chrome em um computador desktop -, quanto por meio do aplicativo da loja, o Play Store - executado num smartphone Samsung Galaxy S8. Esta dupla incursão mostrou-se necessária a partir da observação prévia de uma incongruência na posição de alguns aplicativos entre as recomendações de maior relevância, quando comparados os dois métodos de acesso.

Faz-se aqui necessária uma breve digressão para tornar claro o conceito de relevância no contexto da utilização de motores de busca $^{3}$ online. Silvana Drumond Monteiro e seus companheiros de investigação, ao analisar a estrutura do Sistema de Recuperação de Informação (SRI) do Google como um caso exemplar, argumentam que, atualmente, a relevância nesses sistemas “está associada ao significado, isto é, com a Semântica, conforme descrição dos atributos dos algoritmos do Google" (2017, p. 162). Os autores da pesquisa explicitam que "as palavras semanticamente significativas para a busca são analisadas de acordo com a frequência e localização no documento, análise de links, além de mais outros 200 sinais e 500 aprimoramentos, por ano" (GOOGLE, 2016 apud MONTEIRO et al., 2017, p. 169). Dentre esses aprimoramentos estão, por exemplo, o bummingbird, atualização do PageRank, antigo algoritmo de ranqueamento baseado em métricas de citações; e o Knowledge Graph, um "mapa de elementos do mundo real e as conexões entre eles" (espécie de banco de dados relacional), que tem por objetivo a aprendizagem da máquina e a semantização dos resultados da busca (MONTEIRO et al., 2017, p. 169).

3 “(...) mecanismos de busca, sistemas de busca, motores de busca, buscadores, search engine, são os termos utilizados para indicar um conjunto organizado de computadores, algoritmos, bases de dados e índices reunidos com a função de analisar e indexar as páginas da Web e apresentá-las de forma organizada aos usuários” (Oliveira; Araújo, 2012 apud Monteiro et al., 2017: 165). 
A relevância em um search engine, que busca ser alcançada a partir do tratamento semântico e referencial dos dados, está ligada à tentativa de cumprir totalmente a meta de pesquisa do usuário, ou seja, "a relevância de uma busca só existirá se a ferramenta de busca conseguir, de forma eficaz, identificar o contexto da pesquisa e seus elementos." (FIORAVANTE, 2009 apud MONTEIRO et al., 2017, p. 169). Nesse sentido, o Google utiliza também atributos de personalização, contexto e localização para refinar seu sistema de indexação e busca.

A personalização se faz a partir da intenção de busca, que é inferida por meio das pragmáticas ou trilhas sígnicas semânticas deixadas pelo sujeito navegador, tanto no buscador quanto no ciberespaço. Hoje, é difícil fazer uma "busca pura", isto é, sem algum tipo de personalização. O fato de estar "logado", a cidade em que o usuário se localiza, a plataforma utilizada, o sistema operacional, a navegação, enfim, tudo é capturado por sistemas de busca. Isso quer dizer que as máquinas estão ouvindo o que milhões de pessoas falam, por meio de ferramentas linguísticas e estatísticas (MONTEIRO et al., 2017, p. 171).

Diferentemente de uma busca que abranja toda a rede, procurar por um termo na Google Play Store lida com um contexto de busca e recuperação de dados restritos, por tratar-se de uma plataforma de $e$-commerce em que as próprias companhias desenvolvedoras de softwares submetem seus produtos ao catálogo, ou seja, uma indexação limitada. Ainda assim estão em jogo aí as estruturas de otimização semântica dos dados, de modo a tornar a relevância um atributo da própria informação e buscar apresentar conteúdo significativo para o usuário. Talvez, para maior precisão no cruzamento de dados referentes a uma suposta relevância geral, fosse necessário, no contexto da pesquisa aqui apresentada, ampliar o número de incursões de busca na Google Play Store, utilizando navegadores, sistemas operacionais e dispositivos diferentes - pertencentes a usuários diferentes, em regiões e contextos diferentes. Entretanto, sendo a personalização intrínseca do próprio motor de busca e da ideia de relevância, a pesquisa aqui apresentada está condicionada aos dispositivos e metadados semânticos do autor.

Ao realizar o levantamento dos aplicativos sobre Chapeuzinho Vermelho, buscou-se delimitar o universo de observação aos cinquenta (50) aplicativos mais relevantes - excluindo-se repetições - no resultado da pesquisa pelo termo "red riding hood" através do motor de 
busca da Google Play Store, na categoria $A P P^{4}$. A decisão pelo termo de busca - um dos títulos mais recorrentes para o conto quando em língua inglesa - foi tomada a partir da observação prévia da absoluta maioria quantitativa de aplicativos em inglês, disponíveis na loja online ${ }^{5}$. A decisão de suprimir o artigo "the" e o adjetivo "little" deve-se à necessidade de englobar o maior número de obras na pesquisa; tanto aquelas que possuem tais palavras no título, quanto as que não as possuem.

A partir do universo delimitado de aplicativos, sucedeu-se à classificação em tipologias, levando em consideração as suas principais funções e características formais. Para chegar a essas definições, além de terem sido observados vídeos de utilização disponibilizados pelos produtores (na própria Google Play Store), os aplicativos também foram testados no smartphone. Desse modo, foram classificados nas seguintes categorias: (i) referência à forma do livro ilustrado impresso, com narrativa linear; (ii) referência à forma do livro ilustrado impresso, com narrativa multiforme; (iii) características formais específicas do digital, com narrativa linear; (iv) características formais específicas do digital, com narrativa multiforme; (v) puzzles, arcades, etc; (vi) outros. A tabela com todos os dados do levantamento e classificação segue abaixo:

4 O levantamento foi realizado no dia 13 de outubro de 2018, através de uma rede de acesso particular à internet em Coimbra, Portugal.

5 Além da razão explicitada no texto, é preciso enfatizar que muitos metadados e tags de informações e arquivos online são muitas vezes incluídos em inglês, o que explica, por exemplo, a presença de aplicativos com títulos originais em português, espanhol, holandês, grego e coreano. 


\begin{tabular}{|c|c|c|c|c|}
\hline Categorias & Website & $\begin{array}{l}\text { PlayStore } \\
\text { App }\end{array}$ & Título & Produtora \\
\hline \multirow{27}{*}{$\mathrm{i}$} & 4 & 3 & Little Red Riding Hood Book & TabTale \\
\hline & 13 & 4 & The Little Red Riding Hood & AmayaKids \\
\hline & 18 & 6 & Little Red Riding Hood & Shinypix \\
\hline & 19 & 13 & The Little Red Riding Hood & Active Panda \\
\hline & 29 & 8 & Little Red Riding Hood & Televisor $\mathrm{Sp}$ \\
\hline & 17 & 24 & Little Red Riding Hood ${ }^{1}(\$)$ & $\begin{array}{l}\text { Enjoy Gaming } \\
\text { Ltd }\end{array}$ \\
\hline & 21 & 16 & Little Red Riding Hood $^{1}$ & Mammoth Games \\
\hline & 15 & 49 & Grimm's Red Riding Hood (\$) & StoryToys \\
\hline & 20 & 11 & Little Red Riding Hood Lite ${ }^{1}$ & KD Interactive \\
\hline & $53^{* *}$ & 28 & Little Red Riding Hood Lite ${ }^{1}(\$)$ & KD Interactive \\
\hline & 16 & 26 & Chapeuzinho Vermelho & HippoBaby Game \\
\hline & 27 & 15 & Little Red Riding Hood & Q.L.L. Inc. Ltd. \\
\hline & 26 & 27 & Chapeuzinho vermelho (free) & FORii \\
\hline & 32 & 21 & Little Red Riding Hood & Thematica \\
\hline & 31 & 23 & Little Red-Riding Hood Tale & Codore \\
\hline & 34 & 25 & Little Red Riding Hood & mLabECA \\
\hline & 25 & 41 & Red Hood Ebook & Fun\&Good \\
\hline & 36 & 35 & Little Red Riding Hood Story 2018 & Devfoxmy \\
\hline & 39 & 40 & Chapeuzinho Vermelho conto int. & Rapeto Apps \\
\hline & 49 & 37 & Caperucita Roja & Ikarix \\
\hline & 48 & $\mathrm{X}$ & Red Riding Hood & Komakuro \\
\hline & $\mathrm{X}$ & 48 & Sprookjes van Grimm & RicApps \\
\hline & $78^{*}$ & 46 & $\mu$ & bitAR \\
\hline & $56^{*}$ & 47 & Fairytale - Red Riding Hood (\$) & Zany Studio \\
\hline & $51^{* *}$ & $65^{*}$ & Chapeuzinho Vermelho - conto (\$) & Chocolapps \\
\hline & $54^{* *}$ & $52^{* *}$ & L.R.R.H. - Tales \& livro interativo & Isaballos Apps \\
\hline & $52^{* *}$ & $59^{*}$ & Story For Kids & Touchzing Media \\
\hline \multirow{3}{*}{ ii } & 9 & 10 & R.R.H. int. game story free tale & Living a Book \\
\hline & 14 & $\mathrm{X}$ & Return of Red (Riding) Hood & NBL project \\
\hline & 22 & 31 & Little Red Riding Hood Jarnaby & UI-topias Labs \\
\hline iii & $73^{*}$ & 43 & La Caperucita Roja & PlelQ Stories \\
\hline \multirow[b]{2}{*}{ iv } & 5 & 9 & Red Riding Hood & Sonnar Interactive \\
\hline & 42 & 34 & Little Red Riding Hood & Ubiplay Mobile \\
\hline
\end{tabular}




\begin{tabular}{|c|c|c|c|c|}
\hline \multirow{19}{*}{$\mathrm{v}$} & 1 & 1 & TA: Little Red Riding Hood (\$) & AppGames.net \\
\hline & 3 & 7 & $\begin{array}{l}\text { Cruel Games:Red Riding Hood }{ }^{1} \\
(\$)\end{array}$ & Alawar Entertain. \\
\hline & 37 & $64^{*}$ & Cruel Games:Red Riding Hood ${ }^{1}$ & Alawar Entertain. \\
\hline & 10 & 5 & Red Riding Hood Grimm Slot & Beats'n'Bobs \\
\hline & 8 & 12 & Capuchinho Vermelho: Igualar & Go Vuzzle \\
\hline & 12 & 19 & Red Riding Hood: Corrida 3D & TnTn \\
\hline & 6 & 33 & Night of the full moon & Giant Network \\
\hline & 11 & 17 & Little Red Riding Hood & Interesting games \\
\hline & 7 & 22 & Red Riding Hood (full) (\$) & Anuman \\
\hline & 23 & 50 & Bebê Red Riding Hood Cuidados & Glitter Cute \\
\hline & 28 & 54 & Jungle Red Riding Hood Worlds & NikoDev \\
\hline & 30 & 29 & Slot - Little Red Ridinghood & Fun Casino Game \\
\hline & 35 & $67^{*}$ & Red Hood: Kids English & Fun\&Good \\
\hline & $56^{*}$ & 36 & Little Red Hood Rolling Ball & Tuffyz \\
\hline & 38 & 45 & Little Red Riding Hood Jigsaw & Codore \\
\hline & 40 & 39 & L.R.R.Hood's Forest Adventures & QDLearn \\
\hline & 43 & 42 & Collapsed Little Red Riding Hood & Goog-inc \\
\hline & 44 & 38 & Bring me Cakes - Jogo Fábula & Potato Jam \\
\hline & 47 & $76^{*}$ & Red R. Hood Jigsaw Puzzle Game & daamDAAM \\
\hline \multirow{8}{*}{ vi } & 2 & 2 & Mini Tale Chapeuzinho Vermelho & Mini Tale \\
\hline & 24 & 18 & & Flash\&FirstFox \\
\hline & 33 & 14 & Little Red Hood - Free $^{1}$ & Milk palette+ \\
\hline & 45 & 30 & Little Red Hood $^{1}(\$)$ & Milk palette+ \\
\hline & $57^{*}$ & 32 & Little Red Bow-Red Riding Hood & App. for Android \\
\hline & 41 & 20 & Song for Kids: L. R. Riding Hood & Devfoxmy \\
\hline & 50 & 44 & Little Red Riding Hood (\$) & busythings.co.uk \\
\hline & 46 & $\mathrm{X}$ & Cute Theme-Red Riding Hood & +Home by Ateam \\
\hline
\end{tabular}

Legenda:

(i) referência à forma do livro ilustrado impresso, com narrativa linear

(ii) referência à forma do livro ilustrado impresso, com narrativa multiforme

(iii) características formais específicas do digital, com narrativa linear

(iv) características formais específicas do digital, com narrativa multiforme

(v) puzzles, arcades, etc

(vi) outros

(*) Não estão entre os cinquenta (50) mais relevantes em uma das buscas.

$\left.{ }^{(*}\right)$ A lista real dos aplicativos mais relevantes vai até a posição cinquenta e quatro

(54), dada a existência de versões duplicadas (paga e gratuita).

(X) Não estão nem mesmo entre os cem (100) resultados mais relevantes. 
(\$) Aplicativo pago.

(1) O mesmo aplicativo, em versão paga e gratuita.

Tabela: Posições de relevância das recomendações da busca na Google Play Store.

Janet H. Murray utiliza o termo "multiform story" para descrever "a written or dramatic narrative that presents a single situation or plotline in multiple versions, versions that would be mutually exclusive in our ordinary experience" (2016, p. 37). De forma semelhante, Stavros Halvatzis aplica o conceito de "multiforme narratives" à análise de obras do cinema hollywoodiano, utilizando-o para definir os roteiros cinematográficos com mais de uma versão de desenvolvimento do enredo ou de um acontecimento específico, de modo que ambos os eventos não possam estar em uma única estrutura espaço-temporal: "a paradox that can only be resolved if we place the two events in separate realities" (2015, online). O conceito de narrativa multiforme foi, portanto, aplicado ao contexto deste artigo para definir os aplicativos que organizam a experiência do storytelling de modo a permitir a exploração de realidades diferentes, seja no desenvolvimento ou desfecho das histórias.

A preferência pela utilização do conceito de narrativa multiforme, em vez de história multiforme, foi feita levando em consideração uma distinção técnica bastante refinada entre os dois termos - narrativa e história -, empreendida por Marie-Laure Ryan no verbete "Narrative", presente na "Routledge Encyclopedia of Narrative Theory". Entre outras considerações, Ryan cita H. Porter Abbott, que compreende narrativa como:

the combination of story and discourse and defines its two components as follows: 'story is an event or sequence of events (the action), and narrative discourse is those events as represented' (2002: 16). Narrative, in this view, is the textual actualisation of story, while story is narrative in a virtual form. If we conceive representation as medium-free, this definition does not limit narrativity to verbal texts nor to narratorial speech acts. (...) but this characterisation ignores the fact that events are not in themselves stories but rather the raw material out of which stories are made. (...) Story is a mental image, a cognitive construct that concerns certain types of entities and relations between these entities (RYAN, 2005, p. 347). 
Considerando, portanto, a narrativa como um texto semiótico produzido em qualquer meio, que materialize e seja capaz de evocar uma construção mental (história), distinguindo o discurso narrativo de outros tipos de textos, é possível dizer que alguns aplicativos aqui analisados são exemplos de narrativas multiformes, visto que evocam imagens mentais de realidades diversas, de acordo com as possibilidades programadas. A narrativa multiforme, embora diferente da narrativa linear, não equivale a um rompimento total com a linearidade -, pois apresenta diversos percursos narrativos, cada qual com desenvolvimentos e/ou fechamentos específicos para o enredo. Uma narrativa não-linear, em contrapartida, pode apresentar inversão da ordem dos eventos e até certa indeterminação entre o começo e o fim de uma história.

Diferentemente de algumas configurações de narrativas multiformes, nos aplicativos geralmente não temos acesso direto a todas as realidades possíveis para o desenvolvimento do enredo, apesar sabermos que elas existem. As possibilidades estão condicionadas às escolhas ativas do usuário, ao qual é dado um único caminho como resultado de cada escolha. Se quiser descobrir as outras possibilidades programadas no aplicativo, muitas vezes o usuário deve retornar a uma etapa anterior da narrativa ou recomeçá-la, se possível. Por essas características, a narrativa multiforme nos aplicativos aproxima-se também do conceito de "multi-path narrative": "[a] work of fiction or video/film where the audience or reader at specific points has to choose between branching alternatives in the text" (AARSETH, 2005, p. 323).

Conforme a definição de Espen Aarseth permite observar, este tipo de narrativa, assim como a narrativa multiforme, não é específica de um só meio. Entretanto, Janet Murray percebeu na tecnologia computacional, e nos produtos culturais gerados por ela, a maior possibilidade de mudança nos princípios básicos da estrutura narrativa. "Not only is the computer the most capacious medium ever invented, but it also allows us to move around the narrative world, shifting from one perspective to another at our own initiative" (MURRAY, 2016, p. 261). Hiperficção, jogos eletrônicos, vídeos interativos, livros digitais e aplicativos literários são alguns dos produtos culturais que podem ser citados como exemplos de que "digital formats can provide an expansion of our storytelling powers", de acordo com Murray (2016, p. 263).

O fator fundamental para delimitar o corpus final da análise dos aplicativos de Chapeuzinho Vermelho encontrados pelo sistema de busca é a ênfase na intenção narrativa. $\mathrm{Na}$ fase de classificação, essa foi uma das principais questões observadas: estão os aplicativos a utilizar 
o conto de Chapeuzinho Vermelho apenas como elemento ilustrativo para outro fim; ou uma das finalidades - quiçá a principal - do aplicativo é recontar uma versão da história (literary app)? Tomou-se por base este questionamento para chegar a cinco (5) aplicativos exemplares, especificamente os mais relevantes nas categorias (i), (ii), (iii) e (iv). Os puzzles e arcades (v) ou outros tipos de aplicativos (vi) foram excluídos da análise $e^{6}$. Adiante os aplicativos selecionados serão descritos com maior detalhamento, evidenciando-os enquanto "digital literary art form that combine media affordances and multimodal configurations from different narrative forms" (FREDERICO, 2017, p. 43).

\section{Formas de atravessar a floresta}

"The Little Red Riding Hood" (AmayaKids) ${ }^{7}$ é um claro exemplo de referência à estrutura do livro ilustrado impresso. O aplicativo cria a simulação de um livro sobre a mesa, no qual o usuário pode folhear as páginas repletas de texto e ilustrações com um simples movimento do dedo sobre a tela (swipe). A manutenção da configuração do livro impresso pode ser observada, inclusive, na presença de um sumário. A narrativa do literary app desenvolve-se linearmente e é possível ouvir a narração da história.

Em determinadas páginas prevalecem os elementos visuais e o aplicativo emula o funcionamento de um livro ilustrado pop-up. As ilustrações possuem interações comedidas

6 Na categoria de "puzzles" (quebra-cabeças) e "arcades" (jogos baseados na destreza do utilizador) estão os aplicativos que têm primordialmente função de jogabilidade e entretenimento, utilizando Chapeuzinho Vermelho apenas como referência temática. O mesmo acontece com os aplicativos incluídos na categoria "outros", que engloba aplicativos de customização de personagens até utilitários como um relógio digital. Esses aplicativos não apresentavam elementos da história, passagem de tempo ou sucessão de eventos.

7 Incluído na coletânea "Fairy Tales Children's Books, Stories and Games". Disponivel em: $<$ https://play.google.com/store/apps/details?id=com.amayasoft.bookstorem4.en\&hl=pt-PT> Acesso em 29 de dez. 2019. 
e a esfera sonora é bastante complexa, com diálogos entre os personagens e efeitos sonoros. Nessas páginas pop-up, é possível visualizar o ambiente digital em segundo plano, atrás da representação do livro, o que aparenta ser uma biblioteca.

Elementos de interatividade mais complexos aparecem também no contexto das páginas pop-up. São eles: atividades de combinação (drag and drop, como no desafio para fazer um bolo), reconhecimento (como na atividade dos sons dos pássaros ou dos objetos escondidos no jardim da avó), puzzle (como na travessia do caçador pela floresta) ou jogo de pontaria (como na passagem em que o usuário deve tentar deter o lobo). Com relação a esses exemplos de jogos e brincadeiras, é possível dizer que a maioria deles não parece desproposital (com exceção das atividades de reconhecimento), pois estão bem adequados à estrutura narrativa, apoiando os eventos da história, diferentemente do caso a seguir.

Em "Little Red Riding Hood Book" (TabTale) ${ }^{8}$, muitas das mesmas características descritas acima também estão presentes, porém são utilizadas de forma menos efetiva. O aplicativo é organizado como progressão linear de telas, um modelo bastante comum entre literary apps para crianças. Esse tipo de organização mantém praticamente as mesmas características composicionais do livro ilustrado, mesmo que não houvesse a simulação do passar de páginas no momento da interação para trocar de telas.

No aplicativo da TabTale, além de interações completamente supérfluas em cada ilustração da história - abrir e fechar portas, girar um frasco, balançar um pano, etc -, há efeitos sonoros e narração - com a possibilidade de o usuário gravar a própria voz a contar a história. Ainda sobre interações supérfluas, é preciso enfatizar a grande quantidade de atividades extras que esse aplicativo apresenta no menu inicial, que vai desde quebra-cabeças, jogo da memória, até ferramenta de pintura digital. A presença de tantos passatempos, além de aproximar o aplicativo do conceito de livro de atividades, reflete o uso descontrolado de ferramentas de gamificação, bastante comum no contexto dos projetos de aplicativos para crianças.

Alice Wilder, especialista em mídias educativas, em entrevista a Lisa Guernsey e Michael Levine, enfatiza que existe uma grande diferença entre interatividade em qualquer lugar

8 Disponível em: <https://play.google.com/store/apps/details?id=com.tabtale.littleredridinghoo$\underline{\mathrm{d} \& \mathrm{hl}=\mathrm{pt}>}$ 
e o que ela chama de "interactivity on the plot line" (GUERNSEY e LEVINE, 2016, p. 42). A aplicação de elementos interativos intimamente relacionados com a estrutura narrativa e a construção da história serviria como reforço positivo tanto em interações simples quanto desafios mais complexos. Para Marie-Laure Ryan, o projeto de inclusão de interações mais complexas, como os desafios e brincadeiras, deve herdar também um objetivo narrativo, o que pode motivar as interações do indivíduo diante dessas experiências (RYAN, 2015).

Em se tratando de referências à estrutura do livro ilustrado, "Red Riding Hood interactive game story free tale" (Living a Book) ${ }^{9}$, apesar de apresentar a proposta de ruptura com um único caminho narrativo, possuindo mais de um final possível, é o aplicativo - dentre os cinco selecionados - que mais se aproxima formalmente de um livro ilustrado tradicional. Composto apenas por ilustrações estáticas e texto verbal, as únicas interações possíveis consistem numa ferramenta de mudança de cor do background do texto e nas escolhas que devem ser feitas no decorrer da história (branching alternatives), que determinam o caminho de sua progressão. Assim, a técnica usada no aplicativo aproxima-se daquela aplicada inclusive em outros tipos de livros impressos como os "choose-your-own-adventure' detective books" (AARSETH, 2005, p. 323).

"La Caperucita Roja” (PlelQ Stories) $)^{10}$ é um aplicativo que apresenta a história de Chapeuzinho Vermelho, principalmente, por meio de texto verbal e ilustrações estáticas. A história é conduzida por um assistente narrador localizado na parte inferior da interface. Os personagens presentes nas ilustrações também apresentam diálogos através de balões como os das histórias em quadrinhos. Tanto a narração quanto os diálogos possuem faixas de áudio, aliadas ao recurso de text highlighting, "a very common feature that modifies the color of the text in parallel with the narration" (AUTOR, 2018, p. 122).

No contexto da organização formal, a progressão da história ocorre automaticamente na maior parte da experiência com o aplicativo, podendo também ser controlada pelas setas nos cantos inferior direito e esquerdo - no último caso, para retornar. Contudo, determina-

9 Disponível em: <https://play.google.com/store/apps/details?id=com.livingabook.littleRedRidingHood\&hl=pt>

10 Disponível em: <https://play.google.com/store/apps/details?id=com.pleiq.redcap\&hl=pt-PT> 
dos momentos da experiência narrativa apresentam alguns artifícios que os diferenciam dos outros aplicativos. Chama a atenção a utilização de tecnologias de realidade aumentada (AR) para a apresentação de certos personagens e elementos da história (Chapeuzinho Vermelho, o Lobo, a Avó, o Lenhador e a casa). Tal utilização de AR está mais relacionada a uma função descritiva, podendo ser comparada a uma forma de ilustração, com a característica distintiva única de ser tridimensional.

Outro recurso do literary app da PlelQ Stories, este mais interessante para pensar os caminhos narrativos, é a representação da floresta, tratada como um ambiente explorável em $360^{\circ}$. Nesse ambiente, que emula o que acontece, por exemplo, nos aplicativos de realidade virtual (VR), as movimentações do smartphone correspondem às mudanças na perspectiva visual a que o utilizador tem acesso; perspectiva essa compartilhada pela protagonista. Além disso, é possível avançar pelo ambiente e explorar a floresta. Aqui é apresentada uma tarefa ao leitor, que nesse momento controla não só a visão, mas a ação da Chapeuzinho Vermelho: ela pode coletar flores para a avó. Entretanto, a tarefa não está associada a uma meta estabelecida para avançar na história, como em processos de gamificação mais elaborados, envolvendo recompensas ou avanço de níveis. Ao contrário, este recurso tem mesmo relevância para a narrativa. A cada vez que se avança pelo caminho para tentar atravessar a floresta, o Lobo aproxima-se novamente da Chapeuzinho Vermelho e tenta convencê-la a ir colher flores para a avó - um dos motivos recorrentes em reescritas do conto pelo qual a menina se atrasa e o Lobo chega antes na casa da avó. Esse comportamento programado enfatiza as características psicológicas do personagem. No aplicativo da PlelQ Stories, apesar de tantas inovações formais, a história de Chapeuzinho Vermelho progride linearmente até o fim.

"Red Riding Hood" (Sonnar Interactive) ${ }^{11}$ apresenta a união de diversas camadas significativas dentre seus recursos constitutivos - notadamente o texto verbal, ilustrações estáticas, narração e voz dos personagens, text highlighting, efeitos sonoros e música. Contudo, pode-se dizer que são outras características deste aplicativo as mais essenciais à sua compreensão: a utilização de convenções formais de outros tipos de apps aliadas às características estruturais das narrativas multiformes.

11 Disponível em: <https://play.google.com/store/apps/details?id=com.Sonnar.RedRidingHoo$\underline{\mathrm{d} \& h l=\mathrm{pt}-\mathrm{PT}>}$ 
No literary app da Sonnar Interactive, o usuário inicia a história pela perspectiva da Chapeuzinho Vermelho. A história avança por meio de narrações e diálogos dispostos de maneira semelhante aos apps de mensagens (Whatsapp, Telegram, etc), inclusive com a identificação do personagem que escreve/fala através de uma ilustração de perfil. A progressão é automática, mas pode ser interrompida, e, ao fim de certas sequências narrativas apresentam-se alternativas para a escolha da continuação da história, que podem levar a um fim prematuro, ou progredir ainda mais. Esse recurso, em especial, influencia diretamente a compreensão da história, pois cria diversas possibilidades de desenvolvimento e conclusões - as diferentes realidades da narrativa multiforme. Quando o usuário chega ao fim menos trágico possível, é apresentada a ele a alternativa para mudar a perspectiva da história para o ponto de vista do Lobo. Esse artifício de liberar um conteúdo como recompensa está diretamente relacionado à temática da gamificação, além de servir como exemplo de um dos tipos de multi-path narratives descritos por Aarseth, aqueles em que "the user has to find the one right path among many misleading ones”, ou seja, o final menos trágico citado anteriormente (2005, p. 323).

A característica multiforme da narrativa no aplicativo da Sonnar Interactive é ainda mais clara graças à criação de um sistema de visualização da progressão da história, que pode ser acessado através do menu inferior em qualquer momento da utilização do app. Esse sistema de visualização permite ainda que o usuário retorne a um ponto anterior, de modo a rever uma decisão que não o tenha agradado. Nesse sentido, tal sistema é também uma ferramenta de navegação.

\section{Uma digressão útil: parar para colher flores}

Mesmo para o observador ingênuo, parece claro existir uma grande diferença entre uma história contada por meio de um livro e um aplicativo para smartphone. E seguindo as descrições dos aplicativos apresentados anteriormente, a diferença entre cada um deles também se torna evidente. Contudo, para compreender como essas diferenças são capazes de influenciar a própria experiência narrativa de uma história, alguns referenciais teóricos precisam ser apresentados. São eles os conceitos de interface, cognição incorporada/distribuída e materialidade formal. 
De forma ampla, Jason Nelson, pesquisador e criador de poesia digital, define interface como "a conceptual (as well as physical, electric, digital, or graphical) meeting place, a moment of translation between one entity and another" (2016, p. 136). A partir dessa definição é possível classificar como interfaces tanto um livro impresso, os dispositivos de hardware e o próprio conjunto de comandos e estruturas que configuram um software. Especificamente em relação à interface digital, o poeta a define como "those code-created (either software-built or hand-typed) elements that allow the user/reader to interact with and experience [its] poetic contente" (NELSON, 2016, p. 136). Tal definição engloba tanto elementos básicos como botões, links e animações que respondem a um clique, quanto elementos visuais e interativos mais complexos.

É importante lembrar, entretanto, que Jason Nelson enfatiza que a interface é mais do que apenas um veículo de acesso ao conteúdo; trata-se de um componente fulcral tanto para a criação quanto para a leitura das experiências digitais que agregam textos verbais, imagens, sons, etc. Aplicando ao seu objeto de estudo, Nelson argumenta que "[d]epending on the interface developed and how it is utilized, the digital poem changes shape, reconfigures meaning and becomes an interactive and responsive poetic/fictional creature" (2016, p. 136). Por extensão da definição, é seguro afirmar que não só a interface da poesia digital tem esse papel crítico, mas qualquer interface das experiências englobadas na ampla categoria da electronic literature $(e-l i t)^{12}$, por exemplo, os literary apps.

A importância fundamental da interface para a configuração do objeto literário digital, e, mais precisamente, no seu papel central para o processo de significação, pode ser descrita por meio dos conceitos de cognição incorporada e distribuída.

As ciências cognitivas, como campo interdisciplinar, historicamente desenvolveram perspectivas diferentes para explicar como a cognição humana é formada e determina nossa compreensão do mundo. A perspectiva incorporada (embodied cognition) apresenta a premissa de que o corpo (natureza biológica do homem) influencia diretamente o pensamento: "cognition is situated in the interaction of body and world, dynamic bodily processes such as motor

12 A abrangência de formas e tipos de experiências literárias definidas no termo electronic literature pode ser observada através do site da Electronic Literature Organization. Disponível em: <https://eliterature.org/>. Acesso em 29 de Dezembro de 2019. 
activity can be part of reasoning processes" (HUTCHINS, 2010, p. 428). Dessa maneira, a ação sobre o mundo e a percepção que temos dele não são consideradas separadamente, mas inextricavelmente ligadas entre si e a outros processos cognitivos. Entretanto, não só as capacidades específicas do corpo humano estão em causa no processo de interação com o mundo. As características do mundo também influenciam a percepção e o raciocínio. O homem age sobre o mundo e o mundo age sobre ele num processo retroativo.

Diferentemente de algumas correntes da filosofia da ciência cognitiva, que explicam a cognição em termos de processos que agem somente dentro da mente, a perspectiva distribuída (distributed cognition) enfatiza que a cognição humana é mais bem compreendida como fenômeno em que os mais diversos fatores ambientais e artefatos externos possibilitam operações cognitivas fora dos limites do corpo do indivíduo, sendo eles considerados "artefatos cognitivos" (NORMAN, 1993). Os artefatos cognitivos modificam o processo de organização de habilidades funcionais, formando sistemas cognitivos diferentes daqueles que estariam operando numa atividade em que eles não fossem utilizados (HUTCHINS, 2001). Por exemplo: é possível realizar cálculos confiando puramente no raciocínio, utilizando lápis e papel ou até uma calculadora - cada uma dessas tarefas organiza diferentes sistemas cognitivos para um mesmo fim. De forma geral, os artefatos cognitivos atuam na eficiência da solução de problemas ao reduzir o custo cognitivo de uma operação, aumentar a precisão de uma tarefa ou permitir novas capacidades que seriam improváveis sem o uso do artefato em causa. A fusão de matrizes sociotecnológicas e organismos biológicos leva a mudanças cognitivas nas quais a estrutura de funcionamento efetiva da mente humana é transformada (CLARK, 2003).

No tratamento dado aos conceitos de cognição incorporada e distribuída, a ênfase no corpo humano e nas suas relações com o mundo material é evidente. Entretanto, no senso comum, ao abordar o tema das mídias digitais, em geral tem-se a ideia da imaterialidade, de uma certa intangibilidade. Se a cognição incorporada e distribuída pressupõe uma interação material com o mundo, seria apenas a materialidade do dispositivo (bardware) - mas não a interface - o que influenciaria a experiência do usuário com os literary apps? Essa perspectiva teórica vai de encontro às ideias de Jason Nelson?

Ao analisar o extenso trabalho de Andy Clark sobre o tema, as respostas são claramente negativas. $\mathrm{O}$ autor considera também as construções do raciocínio abstrato humano - con- 
ceitos sociais, políticos e culturais - como estruturas que agem sobre processos cognitivos. A construção desses conceitos só fora possível graças à capacidade de fixação da linguagem: “[t] he human capacity for advanced, abstract reason owes an enormous amount to the way these words and labels act as a new domain of simple objects on which to target our more basic cognitive abilities" (CLARK, 2003, p. 71). Ou seja, aquilo que podemos definir como artefato cognitivo não se trata apenas de um objeto ou ferramenta, mas de qualquer constructo humano criado para resolver um estado-problema; por exemplo, a própria percepção do mundo, seja ele real ou ficcional. Clark enfatiza que, em diferentes momentos, certas formas de materialização criaram diferentes estruturas à percepção: “[p]ictures and spoken words, then written words and diagrams, and most recently the full firepower of interchangeable digital media rank high among the tools by which we press maximum problem-solving power from brains like ours" (CLARK, 2003, p. 75).

Ainda que as perguntas anteriores sejam analisadas no contexto das mídias digitais, é certo que a interface do aplicativo não deve ser excluída da dimensão material da interação. Para compreender a questão deve-se considerar o conceito de materialidade formal, introduzido por Matthew Kirschenbaum (2008). Ao se perguntar sobre em que consistiria a materialidade das mídias digitais, Kirschenbaum elaborou a distinção entre materialidade forense e formal - compreendidas uma em relação à outra.

(...) forensic materiality rests upon the principle of individualization (...) we find forensic materiality revealed in the amazing variety of surfaces, substrates, sealants, and other mate'riel that have been used over the years as computational storage media, and in the engineering, ergonomic, and labor practices that attend computation (KIRSCHENBAUM, 2008, p. 10).

Ou seja, materialidade forense está ligada às estruturas físico-químicas e componentes eletrônicos dos dispositivos computacionais. Entretanto, nesses dispositivos, o usuário está o tempo todo manipulando símbolos, em vez de matéria. $\mathrm{O}$ ambiente digital age como uma projeção abstrata que se sustenta pela capacidade de o dispositivo propagar a ilusão: a página estática de um texto digital num software de leitura em nada corresponde à realidade processual ininterrupta da Random Access Memory que mantém o software a rodar - metaforicamente, no 
sentido de funcionar, no vocabulário da informática - enquanto roda também - realmente -, o hard disk ${ }^{13}$, no processo recorrente de gravação e recuperação de dados em um computador.

Formal materiality is (...) a way of articulating a relative or just-in-time dimension of materiality, one where any material particulars are arbitrary and independent of the underlying computational environment and are instead solely the function of the imposition of a specific formal regimen on a given set of data and the resulting contrast to any other available alternative regimens (KIRSCHENBAUM, 2008, p. 13).

A materialidade formal é, portanto, a materialidade das interfaces digitais, retomando aqui, Jason Nelson: uma configuração codificada que organiza os dados - o texto, as ilustrações, os sons, etc - por meio de um regime formal específico, modificando assim o seu significado. Desta maneira, pode-se afirmar que softwares, como os literary apps possuem uma materialidade específica - a formal. Em conjunção com a materialidade forense dos dispositivos de leitura, esses aplicativos literários exercem bastante influência nas possibilidades de se construir uma narrativa, podendo os produtores manterem-se mais fiéis aos paradigmas dos meios anteriores ou incorporarem novas possibilidades específicas das ferramentas atuais. Dessa maneira, as tecnologias e interfaces historicamente desenvolvidas para contar histórias - como o códex e o livro ilustrado -, bem como os literary apps com todos os seus recursos e diferentes configurações possíveis, podem ser definidos como meta-artefatos cognitivos, já que criam possibilidades diferentes para contar histórias e devem influenciar criticamente o processo cognitivo envolvido na compreensão dessas histórias.

\section{Perder-se é a forma de se encontrar}

As principais características dos literary apps que recontam Chapeuzinho Vermelho aqui analisados, questões centrais sobre as quais este artigo buscou tratar, foram as diferenças

13 Muitos dos sistemas computacionais recentes não utilizam mais dispositivos de memória não-volátil como o HD, que possui um disco giratório em seu interior, mas sim SSD (Solid-State Drive). A menção a esse tipo de memória favorece aqui a metáfora em questão. 
entre a organização formal de cada aplicativo - com referência ao livro ilustrado ou específica das interfaces do ambiente digital - e suas estruturas narrativas - linear ou multiforme.

Com o levantamento realizado na Google Play Store, é possível afirmar que os aplicativos que mantêm características formais em comum com o livro ilustrado são a grande maioria encontrada dentre os cinquenta mais relevantes no resultado da busca realizada na loja online. Esses aplicativos, apesar de manterem tais características de organização do conteúdo, que por vezes beira a tentativa de emulação, apresentam outros recursos multimídia e interativos, como os jogos e desafios - que representam uma aproximação a outras linguagens digitais, como a dos videogames. Decerto nem sempre a utilização desses recursos é feita privilegiando-se a narrativa.

Dentre os exemplos de literary apps com características formais mais associadas ao digital, "La Caperucita Roja" (PlelQ Stories) foi o aplicativo com usos mais inovadores e diversificados das ferramentas do smartphone, aplicando inclusive a realidade aumentada e uma forma de realidade virtual. Já "Red Riding Hood" (Sonnar Interactive) foi o mais inovador em estrutura narrativa, adaptando certas convenções dos aplicativos de trocas de mensagens, associado à estrutura de um choose-your-own-adventure book, além de um esquema de visualização e navegação pela narrativa.

Após uma extensa parametrização do estudo e considerações sobre conceitos teóricos, é possível afirmar que os dois aplicativos supracitados se destacam enquanto experiências narrativas particulares graças às suas materialidades formais exclusivas, capazes de possibilitar percursos cognitivos bastante diferentes daqueles que seriam formados na leitura do conto de Chapeuzinho Vermelho em um aplicativo mais vinculado aos paradigmas do livro ilustrado, por exemplo.

Janet Murray, em sua obra "Hamlet on the Holodeck" (1997, e versões atualizadas em 2016 e 2017), reconhece a existência de histórias multiformes em diversas mídias, mas enfatiza que através das possibilidades trazidas pelo ambiente digital alcança-se a excelência na construção de enredos bifurcados e permutações narrativas em cascata, que expressam uma percepção da realidade característica da contemporaneidade:

Multiform narrative attempts to give a simultaneous form to these possibilities, to allow us to hold in our minds at the same time multiple contradictory alternatives. 


\begin{abstract}
Whether multiform narrative is a reflection of post-Einsteinian physics or of a secular society haunted by the chanciness of life or of a new sophistication in narrative thinking, its alternate versions of reality are now part of the way we think, part of the way we experience the world. To be alive in the twentieth century is to be aware of our alternative possible selves, of alternative possible worlds, and of the limitless intersecting stories of the actual world (MURRAY, 2016, p. 43).
\end{abstract}

Já avançando para o século XXI, a consciência das possibilidades do indivíduo e das construções diversas da realidade parece cada vez mais potencializada. O conto de Chapeuzinho Vermelho coleciona reinterpretações regionais e também aquelas conscientes de questões ambientais e feministas, por exemplo ${ }^{14}$. Apesar de, historicamente, o conto da menina que atravessa a floresta já ser reconhecidamente marcado por reinterpretações e reescritas de autores ao redor do mundo, as possibilidades de perspectiva caleidoscópica que o meio digital apresenta potencializam o papel do leitor, que pode, por exemplo, mudar a perspectiva da história para o ponto de vista do Lobo, como no aplicativo da Sonnar Interactive.

Quantitativamente, os resultados atuais ainda mostram que o caminho pela floresta continua bastante direto (narrativa linear), mas, por vezes, com muitas distrações pelo caminho (multimídia e interatividade mal aplicadas). Entretanto, o futuro mais promissor para Chapeuzinho Vermelho nos literary apps parece ser perder-se cada vez mais por entre boas distrações (interatividade com responsabilidade narrativa) e caminhos diversos (narrativa multiforme), deixando que cada Chapeuzinho siga seu próprio caminho.

14 A título de exemplificação, em “O Capuchinho Vermelho: um livro pop-up”, versão reescrita por Louise Rowe (Portugal: Editorial Presença, 2010), o lenhador sacode o lobo para que o animal cuspa a menina e a avó - em vez de abrir a sua barriga com um machado. Depois o animal vai embora, adentrando novamente o bosque. Na adaptação “The Werewolf”, feita por Angela Carter, escritora inglesa pós-modernista de reconhecida expressão feminista, Chapeuzinho Vermelho ataca o lobo quando ele aparece para assustá-la na floresta. Mais tarde, descobre ser o lobo a própria avó, cuja morte pode simbolizar a metamorfose da puberdade, da neta que toma o lugar da avó. O texto de Carter pode ser encontrado em "The bloody chamber" (London: Vintage, 1995, p. 108-110). 


\section{Referências:}

AARSETH, Espen. Multi-path narrative. In: HERMAN, D.; JAHN, M.; RYAN, M-L. (Eds.). Routledge Encyclopedia of Narrative Theory. New York: Routledge, 2005, p. 323-324.

CLARK, Andy. Natural-born cyborgs: minds, technologies, and the future of buman intelligence. New York: Oxford University Press, 2003.

ESTEFANI, Thales; QUEIROZ, João. Children's picturebook goes digital: implications on cognition. In: $\quad$ MATLIT: Materialidades da Literatura, Coimbra: 6 (2): 115-127, 2018.

FREDERICO, Aline. Children making meaning with literary apps: a 4-year-old child's transaction with The Monster at the End of This Book. In: Paradoxa, Vashon Island: 29 (Small Screen Fiction): 41-64, 2017.

GUERNSEY, Lisa; LEVINE, Michael H. Getting smarter about e-books for children. In: Young Children, Washington, D.C.: 38-43, maio 2016.

HALVATZIS, Stavros. What is multiform narrative? Website pessoal, mar. 2015. Disponível em: http://stavroshalvatzis.com/story-design/what-is-multiform-narrative. Acesso em 10 set. 2019.

HUTCHINS, Edwin. Enaction, imagination, and insight. In: STEWART, J.; GAPENNE, O.; DiPAOLO, E. A. (Eds.). Enaction: toward a new paradigm for cognitive science. Cambridge, Massachusetts: The MIT Press, 2010, p. 425-450.

HUTCHINS, Edwin. Cognition, distributed. In: SMELSER, N. J.; BALTES, P. B. (Eds.). International Encyclopedia of the Social \& Behavioral Sciences. Oxford: Elsevier, 2001, p. 2068-2072.

KIRSCHENBAUM, Matthew G. Mechanisms: new media and the forensic imagination. Cambridge, Massachusetts: The MIT Press, 2008. 
MONTEIRO, Silvana D.; FERNANDES, Rogério P. M.; DECARLI, Gian Carlo; TREVISAN, Gustavo L. Sistemas de recuperação da informação e o conceito de relevância nos mecanismos de busca: semântica e significação. In: Encontros Bibli, Florianópolis: 22 (50): 161-175, set./dez. 2017.

MURRAY, Janet H. Hamlet on the Holodeck: the future of narrative in cyberspace (versão atualizada em e-book). New York: The Free Press, 2016.

NELSON, Jason. What is an interface and why does a digital poet care? In: GRAMMA: Journal of Theory and Criticism, Thessaloniki: 23: 136-144, 2016.

NORMAN, Donald. Things that make us smart: defending human attributes in the age of the machine. Boston: Addison-Wesley Publishing Company, 1993.

RYAN, Marie-Laure. Narrative. In: HERMAN, D.; JAHN, M.; RYAN, M-L. (Eds.). Routledge Encyclopedia of Narrative Theory. New York: Routledge, 2005, p. 344-348.

RYAN, Marie-Laure. Narrative as virtual reality 2: revisiting immersion and interactivity in literature and electronic media. Baltimore: John Hopkins University Press, 2015.

SARGEANT, Betty. What is an ebook? What is a book app? And why should we care? An analysis of contemporary digital picture books. In: Children's Literature in Education, Springer Netherlands: 46: 454-466, 2015.

STICHNOTHE, Hadassah. Engineering stories? A narratological approach to children's book apps. In: Nordic Journal of ChildLit Aesthetics, Oslo/Londres: 5 (1), artigo 23602, 2014. 